\title{
Research Reporting Guidelines and the New Annals Instructions for Authors
}

\author{
Aaron M. Orkin, MD, MSc, MPH \\ William R. Pbillips, MD, MPH \\ Kurt C. Stange, $M D, P b D$ \\ Ann Fam Med 2016;14:500-501. doi: 10.1370/afm.2008.
}

W ith this issue, Annals of Family Medicine introduces our updated Instructions for Authors. We aim to help authors report their best work in ways that are most useful to readers. The updated Instructions for Authors provide enhanced access to reporting guidelines as tools to support best practices in reporting health research. We believe that thoughtful use of these reporting guidelines will help advance the quality, application, and impact of research in primary health care.

High quality reporting guidelines are now widely accepted for most health research methods, including qualitative, quantitative, and mixed methods research, as well systematic reviews, quality improvement, and program evaluations. A checklist accompanies most reporting guidelines, making them easy for authors to use and cite.

Many papers that we receive are a good fit for one or more of these guidelines. We encourage authors to use the reporting guideline appropriate for their study design and to submit the checklist with their manuscript.

The Instructions for Authors provide direct links to common reporting guidelines and to the EQUATOR Network, an international initiative that seeks to improve the reliability and value of published health research by promoting transparent and accurate reporting and wider use of robust reporting guidelines. The EQUATOR website serves as a clearing-

Conflicts of interest: At the time this was written, Dr Orkin was the Editorial Fellow and Drs Phillips and Stange are editors at the Annals of Family Medicine.

\section{CORRESPONDING AUTHOR}

Aaron Orkin, MD, MSc, MPH

Schwartz/Reisman Emergency Medicine Institute 600 University Avenue

Toronto, M5G1X5 house of up-to-date reporting guidelines and related tools and publications. ${ }^{1}$

In designing these Instructions for Authors, we followed an inclusive process. We considered feedback from authors and peer reviewers and from discussion in interactive workshops we held on reporting guidelines at the North American Primary Care Research Group 2015 Conference and at the College of Family Physicians of Canada's Family Medicine Forum 2015. We also reviewed best practices from leading journals and considered how the Annals can contribute most to the advancement of research and its clear and useful dissemination.

Even among top-tier journals and papers, serious deficiencies are common in reports of health research across a wide range of methodologies. ${ }^{5-8}$ Low quality research and deficient reporting can generate "research waste," with serious bioethical and economic consequences. ${ }^{9}$ Primary care research is not exempt from these concerns.

Other journals have found that placing more emphasis on reporting guidelines improves methodological transparency, the peer review process, and ultimately the quality of published research. ${ }^{10,11}$ Clinicians have embraced evidence-based medicine and become more critical as they read and appraise research. Reporting guidelines can help assure that primary care research delivers the methodological information that we have been trained to seek as we integrate research findings into clinical practice, and into policy and educational applications.

Reporting guidelines are evolving constantly. Just like a clinical practice guideline may not be right for every patient, reporting guidelines may not be right for every study. The most creative and valuable research often stretches beyond conventional methods. This is especially true for the generalist research that Annals often publishes. If authors consider a relevant guideline 
but decide not to use it, we ask that they offer a brief rationale for this in their cover letter when submitting the manuscript.

Rigor is about critical thinking, sound understanding of methodology, and a logical approach to research questions-not about following a guideline or working through a checklist. Our intent is to support rigor, not to stifle creativity, generate dull manuscripts, or create more work for authors. Reporting guidelines need not result in longer or formulaic manuscripts. The guidelines can help researchers report their methods succinctly, so that other aspects of the paper may flourish. Composing a compelling report still relies on the writer's craft and the story behind the science.

We suggest researchers refer to reporting guidelines early in their work, as they formulate research questions and design and conduct studies. ${ }^{7}$ Guidelines can serve as a roadmap to study and protocol development and help protect against omitting essential methodological detail that might otherwise not come to light until the editorial and peer review process. The SAMPL statistical reporting guidelines can help researchers and biostatisticians ensure that they report their work comprehensively and transparently. ${ }^{12}$

Most of these guidelines emphasize internal validity. We encourage investigators to consider external validity throughout the research process. Identify and report contextual factors that are relevant to understand what happened and why in the study. Help readers to transport and re-invent results in other times and situations. ${ }^{9-11}$

Reporting guidelines and their associated checklists can be useful aids in crafting a report that is complete, efficient, and transparent. Use of a reporting guideline checklist has been shown to improve authors' experience in the peer review process and their ability to respond to reviewer comments. ${ }^{7}$

As editors of Annals of Family Medicine, we are honored to publish some of the best in family medicine and primary care. We believe that judicious use of reporting guidelines can improve the publication experience for all. Reporting guidelines can help us deliver the best of research in the most useful way to clinicians and policy makers and the best of care to the patients and communities they serve.

To read or post commentaries in response to this article, see it online at http://www.annfammed.org/content/14/6/500.

Submitted December 18, 2015; submitted, revised October 14, 2016; accepted October 14, 2016.

Key words: reporting guidelines; scientific writing; primary care research; publishing standards; quality control; epidemiologic research design; guidelines

\section{References}

1. Groenwold RH, Van Deursen AM, Hoes AW, Hak E. Poor quality of reporting confounding bias in observational intervention studies: a systematic review. Ann Epidemiol. 2008;18(10):746-751.

2. Bennett C, Khangura S, Brehaut JC, et al. Reporting guidelines for survey research: an analysis of published guidance and reporting practices. PLoS Med. 2010;8(8):e1001069.

3. Lewin S, Glenton C, Oxman AD. Use of qualitative methods alongside randomised controlled trials of complex healthcare interventions: methodological study. BMJ. 2009;339:b3496.

4. OCathain A. The quality of mixed methods studies in health services research. J Health Serv Res Policy. 2008;13(2):92-98.

5. Chalmers I, Glasziou P. Avoidable waste in the production and reporting of research evidence. Lancet. 2009;374(9683):86-89.

6. Cobo E, Cortés J, Ribera JM, et al. Effect of using reporting guidelines during peer review on quality of final manuscripts submitted to a biomedical journal: masked randomised trial. BMJ. 2011;343:d6783.

7. Medicine Editors PLOS. Transparency in reporting observational studies: reflections after a year. PLoS Med. 2015;12(10):e1001896.

8. The SAMPL Guidelines. In: Smart P, Maisonneuve H, Polderman A, eds. Science Editors' Handbook. European Association of Science Editors; 2013.

9. Stange KC, Glasgow RE. Considering and reporting important contextual factors in research on the patient-centered medical home; 2013. AHRQ Publication No. 13-0045-EF: http://pcmh.ahrq.gov/ page/contextual-factors-importance-considering-and-reportingcontext-research-patient-centered.

10. Tomoaia-Cotisel A, Scammon DL, Waitzman NJ, et al. Context matters: the experience of 14 research teams in systematically reporting contextual factors important for practice change. Ann Fam Med. 2013;11(1):S115-S123.

11. Stange KC. In this issue: practice change-context matters. Ann Fam Med. 2013;11(3):198-199. 